\title{
Fast and Reliable Order Management Design Using a Qualitative Approach
}

\author{
Hans-Hermann Wiendahl \\ Institute of Industrial Manufacturing and Management (IFF), \\ University Stuttgart Nobelstrasse 12, 70569 Stuttgart, Germany \\ Tel: +49(0)711-970-1968, hhw@iff.uni-stuttgart.de
}

\begin{abstract}
Abrupt and often surprising changes characterize the situation of manufacturing companies. Important for an order management design are those factors which potentially cause turbulence and lead to schedule deviations. The paper describes a method to capture and assess them qualitatively. Based on an analogy to physics, the morphology of turbulence germs is provided. Then, a procedure is described how to successfully transfer approaches for turbulence management to other companies. The last part reflects in detail on the application experience.
\end{abstract}

\section{Keywords}

Manufacturing, management, production planning and control, turbulence

\section{Introduction}

Manufacturing companies are faced with changing market and supplier conditions, which greatly affect purchase and production logistics. They apply different manufacturing strategies and organizational changes to cope with these turbulent markets. Rapidly changing logistic conditions require fast and appropriate changes to the existing Order Management (OM) system [1]. Many of those in charge respond in an event-driven manner, involving substantial risks: Either so-called "best practices" are adopted without second thoughts, ignoring necessary requirements, or new solution approaches are developed, without considering logistical interactions.

Experience shows that factors which potentially cause turbulence (i.e. dynamics) are the main challenges for OM design. So it must be possible to quickly and efficiently compare the influence of these factors. The paper introduces a novel method of capturing the influence in a qualitative way and transferring OM solution modules successfully from one company to another. 


\section{Theoretical Fundaments}

The research objective of this paper is to compare the logistical requirements that might cause turbulence in an effective and efficient way. An analogy to physics provides the theoretical fundament of the approach.

\subsection{Definition of Order Management}

Manufacturing companies act in a network of customer-supplier relationships. This requires planning because customers expect reliable delivery promises, while purchase orders need to be placed with the suppliers. The primary order management function is to allocate items, processes and (human and manufacturing) resources to orders in terms of time and quantity [2].

For this purpose, the planning function plans the production flow in advance for a certain period of time, typically based on mean values for lead times. Then, the control function has to implement the plan in the best possible way in spite of changes and disturbances [3]. In doing so, the control function splits up the macroscopic planning results into a microscopic view of single events. Usually, such a plan can never be executed precisely. Therefore - similar to the quality control approach - a tolerance for permissible deviations has to be specified [4].

\subsection{Turbulence}

The definition of turbulence in OM follows physics [5]: Turbulence exists if individual values (microscopic view) deviate significantly from the representative value (macroscopic view of an aggregated value) [6]. To detect a significant deviation, it takes tolerances defining allowed deviations between individual values and the representative, i.e. typically the mean value. These conditions limit the prediction of an individual value by a mean-value based planning approach for reaching the required reliability. As a result, controlling turbulence takes two views $[6,7]$ :

- Objectively, turbulence can be identified by significant deviations - i.e. there are individual values deviating more than allowed from the mean value, Fig.1a.

- Subjectively, turbulence means to have objective turbulence not under control. Comparing required to actual reliability - i.e. requirements compared to capabilities - shows if significant deviations are under control, Fig. Ib.

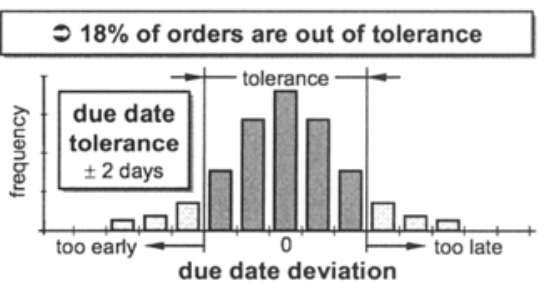

a) objective turbulence

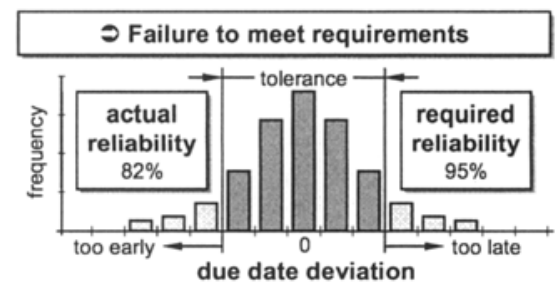

b) subjective turbulence

Fig. 1. Objective and subjective turbulence (example of due date deviation) 


\section{Turbulence Germs in Order Management}

Applying these findings to OM operation, it is necessary to identify the turbulence triggers. The so-called turbulence germs that affect planning and control can be clustered in five groups:

- Relevant for planning are variations, fluctuations and plan adaptations.

- Tolerances define the allowed deviation from planned values. They judge the relevance of a turbulence germ and represent a cause cluster of their own.

- Control deals with unexpected deviations that jeopardize the promised delivery. Note that turbulence germs do not necessarily lead to plan deviations. The relationship between requirements and capabilities determines their relevance, Fig. $2 b$.

OM covers the processes Source, Make and Deliver [8]. Setting them against the five cause clusters will result in the morphology of OM turbulence germs [9], Fig. 2.

\section{Variations}

Variations represent heterogeneous requirements within the same period. The relation between variations resulting from heterogeneous requirements (delivery times, lead times, and replacement times) and permissible deviation (planning tolerance) defines the relevance of turbulence germs.

From a delivery point of view, heterogeneous customer requirements complicate planning: If they lead to heterogeneous order lead times, they will violate the basic assumptions of the successive planning concept MRP II with its mean-based lead time planning approach. If variations exceed planning tolerances, the smooth order flow is disrupted, resulting inevitably in plan deviations. From a make point of view, the necessity of large batches for technological reasons can also create a critical heterogeneous order flow. Heterogeneous replenishment conditions (e.g. Iong-lead items) have a similar effect on sourcing.

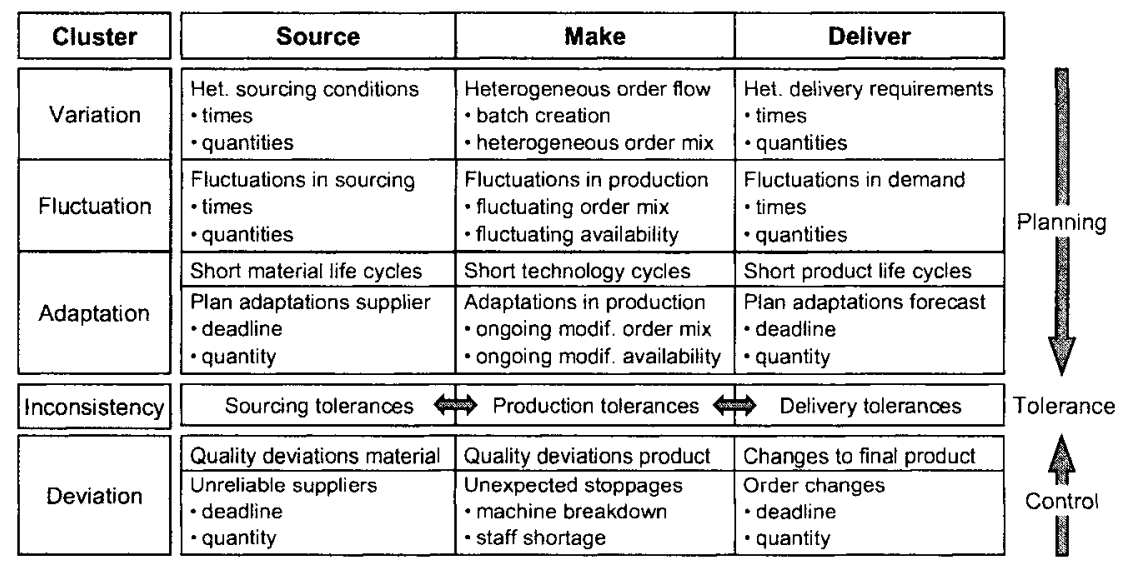

Fig. 2. Morphology of turbulence germs in order management 


\section{Fluctuations}

Fluctuations represent heterogeneous requirements within different periods. Main requirements are the variability of demand in terms of total quantity (delivery view) or order mix (make view): the more varying the quantities in relation to the available quantity flexibility in manufacturing, the greater the turbulence. Fluctuating sourcing conditions (times or quantities) have a similar effect.

\section{Plan adaptations}

Planning should anticipate future. Rolling planning approaches are used to deal with changing delivery and supply situations for the same planning period. That means that depending on the point of time in which the forecast is made, the quantity of the planned period changes. Frequently changing circumstances complicate planning, e.g. short product life cycles or forecast deviations concerning deadline and quantity (deliver), as well as short life cycles of materials and purchased parts or ongoing plan adaptations by the supplier (source). From the make perspective, short technology cycles and ongoing modifications of order mix or resource availabilities (e.g. staff, machine, tools) can cause turbulence.

\section{Inconsistent tolerances}

Logistical requirements also affect the interface between planning and control. From a delivery point of view, the requested delivery tolerances are of particular relevance. For instance: In case of ship-to-stock, it could be sufficient if the delivery arrives anytime within a specified week, while just-in-time deliveries directly to the assembly line must arrive on the exact day or even shift. For differing external requirements, the valid internal planning tolerance must be decided on beforehand. Heterogeneous production or sourcing tolerances complicate planning and control in a similar way.

\section{Deviations}

Deviations from planned values occur when unexpected events after order release necessitate interventions. From a delivery perspective, changes of orders in terms of end product, order quantity or deadline require an intervention by control. From a make perspective, a distinction between unexpected quality deviations, staff shortages and machine breakdowns makes sense. During sourcing, unexpected material deviations (technical specification) and unreliable suppliers can result in missing parts.

\section{Turbulence Profile}

To analyze the impact of turbulence germs in practice, it is advisable to generate a turbulence profile. The investigation is based on a qualitative evaluation of the germs. Application experience from approx. 40 cases shows the practical advantages of a workshop-based procedure to evaluate the germs by technical experts:

1. Explain the theoretical foundation of turbulence and its consequences for OM.

2. Select the relevant germs and judge them individually (by each technical expert).

3. Aggregate the results to create a company-specific turbulence profile, discuss the judgment (similarities, differences) and reasons for the impact of each germ. 


\section{Case Study}

The application is described for a company unsatisfied with its logistical performance. To improve logistics, a two-step procedure was agreed on:

- Firstly, develop an OM design concept. Derive solution blocks and combine them into a consistent approach for OM design, if necessary based on a quantitative assessment of the root causes.

- Secondly, transfer appropriate solution blocks from other companies. Compare the turbulence profiles. Discuss the concept's validity, identify transferable solution blocks and transfer experience.

The following description reflects main discussion points and the OM design consequences for planning and control for one exemplary area.

\subsection{Initial Situation}

The mechanical forming area is characterized by job-shop production with 30 capacity-relevant work centers and 250 workers; work-in-process of 1,800 orders with 11,000 operations in production; simple product structures (1-3 levels). Fig. 3 shows the investigated turbulence profile for this area; on average, planning germs are rated higher: Heterogeneous delivery quantity requirements and demand fluctuations dominate here. From a control point of view, unreliable production processes and deviations from the required material specification are rated highest, since the company works at the cutting-edge of manufacturing technology.

Of course, such a qualitative evaluation does not replace quantitative analyses but reduces effort by focusing on important points. Here, quantitative results confirm the qualitative evaluation and highlight two aspects: The planning approach disregards the manufacturing complexity (changing demand, job shop production) and in addition, planners fix the schedule (firm order quantity and production operation dates) as early as possible. As a result, the control activities after order release cannot implement the fixed schedule within the agreed tolerances.

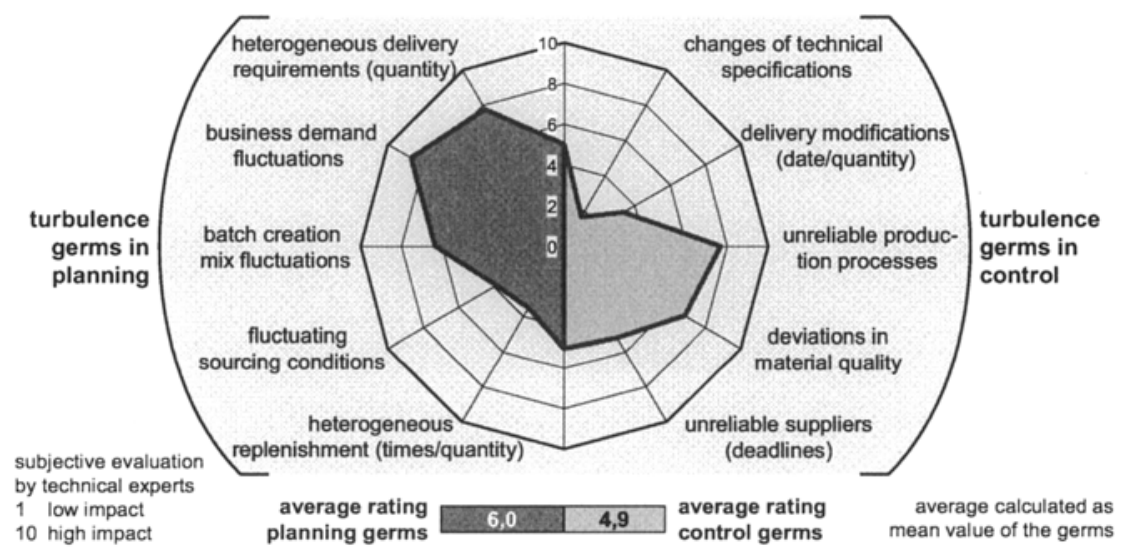

Fig. 3. Turbulence profile company 1, area mechanical forming 


\subsection{Requirements and Solution Blocks for Planning}

Today, planners schedule in a semi-automated way based on the MRP approach; they are supposed to check capacity to avoid an overload. Unrealistic production plans lead to the following questions: Firstly, what level of detail is adequate for planning - i.e. is the detailed scheduling of operations still necessary? Secondly, can the MRP approach with its infinite capacity planning algorithm still be used or is a finite planning approach advisable?

Demand and mix fluctuations get high ratings by the experts (Fig. 3). Detailed quantitative analyses verify that these germs create bottlenecks at different times and places for this job-shop production environment. At first, the idea was to redesign the factory into product segments based on simple material flows. Economic reasons, however, did prevent this lean alternative (due to high investment in new machines). To check for possible bottleneck situations at the work centers, a higher planning complexity must be accepted. This requires the scheduling of operations.

In addition, the capacity planning approach needs to be redesigned: On the one hand, heterogeneous delivery requirements are rated high. Customers order different quantities, resulting in heterogeneous production lot sizes with different capacity demand. On the other hand, the available capacity flexibility is high but differs for each work center in time and quantity. Complex material flows complicate the precise temporal forecast of capacity demand, resulting in high WIP fluctuations; quantitative analyses and discussions with the foremen prove this thesis. A 'double scheduling' approach produces an adequate capacity forecast horizon:

- Purely manufacturing on demand requires infinite flexibility. So, the infinite scheduling run quantifies the capacity demand (quantity and period).

- A realistic plan takes into account finite capacities (and material availability). So, the finite scheduling run forecasts the probable due dates of the orders.

The results of both scheduling runs have to be compared. Deviations from order or resource view point out bottlenecks and indicate the need for planner interventions.

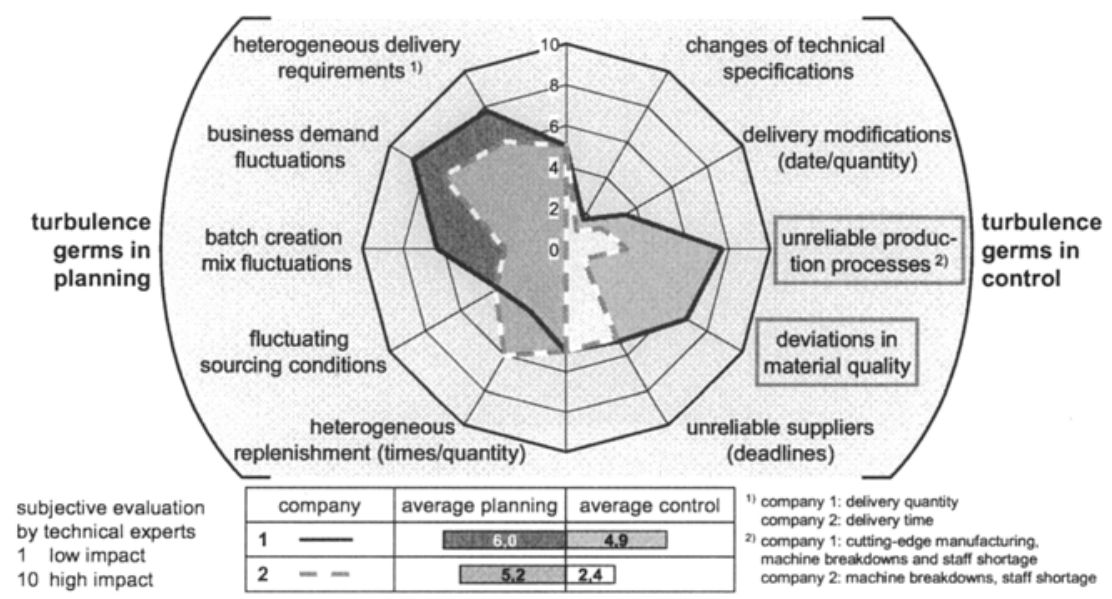

Fig. 4. Comparison of turbulence profiles (company 1 and company 2) 


\subsection{Requirements and Solution Blocks for Control}

Control functions corresponding to the designed planning functions are:

- WIP-oriented order release checking availability of physical material to damp the 'unreliable suppliers' germ and support order execution according to plan.

- Recording of 'sequence discipline' to monitor execution behavior.

More important is another topic: From a control point of view, the above-mentioned 'unreliable processes' and 'material quality deviations' germs get the highest ratings. This advises regular rescheduling, i.e. non-fixed operation dates.

Internal experts fear that this prevents successful software-based OM: Experience shows that ongoing plan changes undermine plan's acceptance. Such a discussion reflects the classical area of conflict between plan stability and dynamics:

- On the one hand, planning should anticipate future. The turbulence analogy shows: The less plans change the more stability - and reliability - they create.

- On the other hand, planning should be up-to-date. If demand changes or a process disturbance occurs, it makes sense to update the old plan. But the turbulence analogy implies that fast changing plans lose people's acceptance.

In this area of conflict, each company has to find its own position. Based on the results, a comparison with two other companies' successful implementations should help to clarify the arguments.

\subsection{Comparison of Turbulence Profiles}

The companies handle turbulence differently: The first executes a fixed schedule with high flexibility; the other achieves acceptance with a not-fixed schedule.

Company 2 produces equipment assemblies. The turbulence profile shows that a highly reliable production allows fixed schedules, Fig. 4. Central planner focus on planning germs and foreman use local flexibility to deal with the control germs. A strict check for material availability supports execution according to plan.

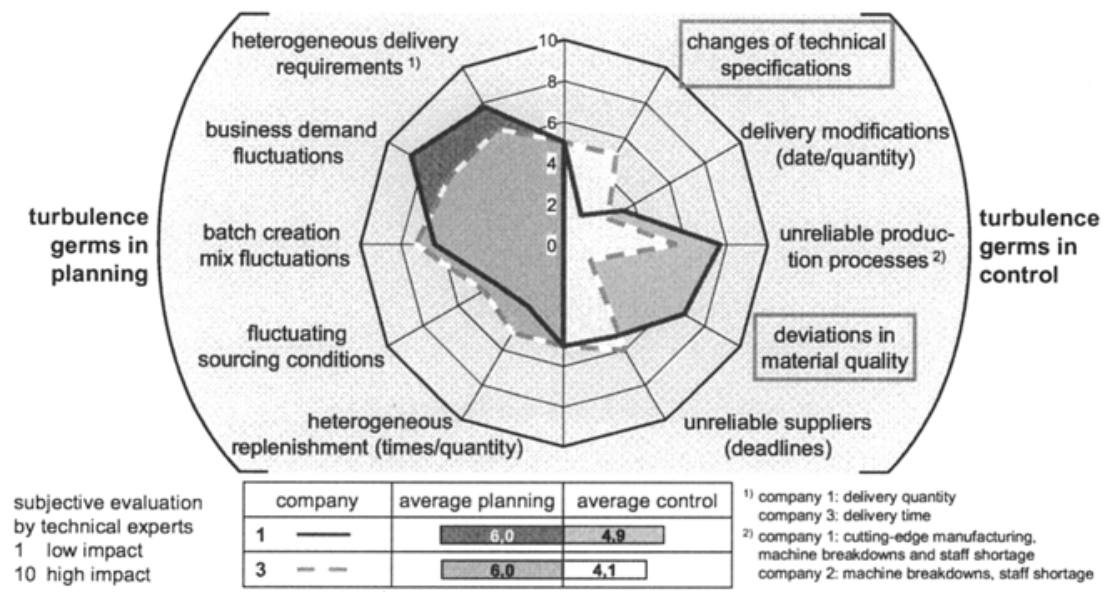

Fig. 5. Comparison of turbulence profiles (company 1 and company 3) 
With the turbulence profile of Company 3 (plant manufacturer), the rating of control germs is higher, Fig. 5. People achieve the plan acceptance in two ways: Firstly, they distinguish between promised, planned and actual dates, visualize the bottlenecks and indicate where planners should take action. Secondly, they split up information into a loading list for 2-3 days (individual orders for work preparation) and the demand information for 4-6 weeks (capacity forecast per week).

The project team decided to integrate the company 3 solution into their concept. It fits their requirements and proved practicable in a comparable situation.

\section{Summary}

Turbulent markets require a fast, effective and efficient order management alignment. The proposed method supports this goal in various ways: It allows the structured qualitative analysis of requirements, derives consistent solutions, and encourages the discussion of logistical interactions and their effects on OM design and operation. The results enable an effective comparison of the logistical requirements and solution blocks from different companies. The outcome accounts for the chosen design solution and hints at possible software tools.

Of course, a qualitative evaluation does not replace quantitative analyses. But the effort for data collection, analysis and verification can be substantially reduced. Experience from about 40 case studies proved the practicability of the approach.

\section{Acknowledgements}

The results are part of the ongoing research project "Model-based Order Management Design for discrete manufacturing" which is funded by the German Research Foundation DFG (WI 2670/1).

\section{References}

1. H.-P. Wiendahl, S. Lutz, Production in Networks, CIRP Annals 51/2, 573-586 (2002).

2. H.-H. Wiendahl, Changeability in Production Planning and Control : A Framework for Designing a Changeable Software Tool, Advances in Production Management Systems: Modeling and Implementing the Integrated Enterprise, edited by IFIP 5.7 (2005).

3. W. J. Hopp, M. L. Spearman, Factory Physics (Irwin, Chicago, 1996).

4. J. Juran, B. Godfrey (ed.), Juran's Quality Handbook (McGraw-Hill, New York, 1999).

5. B. S. Massey, Mechanics of Fluids (Van Nostrand Reinhold, London, 1998).

6. H.-H. Wiendahl, N. Roth, E. Westkämper, Logistical Positioning in a Turbulent Environment, CIRP Annals 51/1, 383-386 (2002).

7. H. Mintzberg, That's not Turbulence, Chicken Little, It's Really Opportunity, Planning Review 11/12, 7-9 (1994).

8. Supply Chain Operations Reference Model (May 12, 2007); http://www.supply-chain.org.

9. H.-H. Wiendahl, Turbulence Germs and their Impact on Planning and Control - Root Causes and Solutions for PPC Design, CIRP Annals 56/1 2007 (to be published). 